TRANSACTIONS OF THE

AMERICAN MATHEMATICAL SOCIETY

Volume 357, Number 11, Pages 4533-4553

S 0002-9947(05)03989-9

Article electronically published on June 21, 2005

\title{
FREE AND SEMI-INERT CELL ATTACHMENTS
}

\author{
PETER BUBENIK
}

\begin{abstract}
Let $Y$ be the space obtained by attaching a finite-type wedge of cells to a simply-connected, finite-type CW-complex.

We introduce the free and semi-inert conditions on the attaching map which broadly generalize the previously-studied inert condition. Under these conditions we determine $H_{*}(\Omega Y ; R)$ as an $R$-module and as an $R$-algebra, respectively. Under a further condition we show that $H_{*}(\Omega Y ; R)$ is generated by Hurewicz images.

As an example we study an infinite family of spaces constructed using only semi-inert cell attachments.
\end{abstract}

\section{INTRODUCTION}

In this article we will work in the usual category of pointed, simply-connected topological spaces with the homotopy type of finite-type CW-complexes. We will assume that the ground ring $R$ is either $\mathbb{F}_{p}$ with $p>3$ or is a subring of $\mathbb{Q}$ which contains $\frac{1}{6}$.

We are interested in the following problem, perhaps first studied by J.H.C. Whitehead around 1940 [Whi41 Whi39].

The cell attachment problem: Given a topological space $X$, what is the effect on the loop space homology and the homotopy type if one attaches one or more cells to $X$ ?

We approach this problem from the point of view that one is interested in understanding finite cell complexes localized away from finitely many primes Ani92.

Given a space $X$ and a map $f: W \rightarrow X$ where $W=\bigvee_{j \in J} S^{n_{j}}$, the adjunction space

$$
Y=X \cup_{f}\left(\bigvee_{j \in J} e^{n_{j}+1}\right)
$$

is a homotopy cofibre of $f$. Let $i$ denote the inclusion $X \hookrightarrow Y$.

The cell attachment problem has been studied in two special cases. One approach is to place a strong condition on the space $X$. This was done by Anick Ani89 who considered the case where $X$ is a wedge of spheres. Another approach is to place a strong condition on the attaching map $f$. This was done by Lemaire and Halperin [Lem78], [HL87] and Félix and Thomas [FT89] who assumed that $f$ is inert.

The attaching map $f: W \rightarrow X$ is said to be inert over a ring $R$ if the induced map $H_{*}(\Omega i ; R): H_{*}(\Omega X ; R) \rightarrow H_{*}(\Omega Y ; R)$ is a surjection.

Received by the editors December 5, 2003.

2000 Mathematics Subject Classification. Primary 55P35; Secondary 16E45.

Key words and phrases. Cell attachments, loop space, loop space homology, Adams-Hilton models, differential graded algebras, Lie models.

(C)2005 American Mathematical Society Reverts to public domain 28 years from publication 
In this article we generalize these two approaches, with our development following [Ani89]. We generalize Anick's assumption to the more general condition that $H_{*}(\Omega X ; R)$ is $R$-free and is generated by Hurewicz images. This is trivial in the case where $R=\mathbb{Q}$, and we will give conditions under which this holds for more general coefficient rings (see Corollary 1.9). Furthermore we give two generalizations of the inert condition, one of which is strictly stronger than the other.

We now define these conditions in the case where $R$ is a field. We will use the following notation.

Notation 1.1. Given a space $X$, let $L_{X}$ denote the image of the Hurewicz map $h_{X}: \pi_{*}(\Omega X) \otimes R \rightarrow H_{*}(\Omega X ; R) . L_{X}$ is a graded Lie algebra under the commutator bracket of the Pontrjagin product. Given a map $W \rightarrow X$, let $L_{X}^{W}$ denote the image of the induced Lie algebra map $L_{W} \rightarrow L_{X}$. Note that the map is omitted from the notation. Let $\left[L_{X}^{W}\right] \subset L_{X}$ denote the Lie ideal generated by $L_{X}^{W}$.

Definition 1.2. Define a cell attachment $f: W \rightarrow X$ to be free if $\left[L_{X}^{W}\right]$ is a free Lie algebra.

There are examples of spaces given by non-free cell attachments that can be constructed by free cell attachments if one changes the order in which the cells are attached (eg. $\mathbb{C P}^{2} \cup_{f} e^{3}$ where $f$ is the inclusion $S^{2} \hookrightarrow \mathbb{C P}^{2}$; see [HL96, Example 4.5]). When $R$ is a field, it is conceivable that any cell complex can be constructed using only free cell attachments if one chooses an appropriate cellular structure. In a subsequent paper [Bub04], the author has shown this is true when $R=\mathbb{Q}$ for spaces with finite rational cone length.

Free cell attachments are convenient to work with because of the following fact about universal enveloping algebras, which we prove as Lemma 3.8. Since it may be of independent interest we state it here. $U$ denotes the universal enveloping algebra functor.

Proposition 1.3. Let $L$ be a connected, finite-type Lie algebra over a field. Let $J$ be a Lie ideal of $L$ which is a free Lie algebra, $\mathbb{L} W$. Take $I$ to be the two-sided ideal of $U L$ generated by $J$. Then the multiplication maps $U L \otimes W \rightarrow I$ and $W \otimes U L \rightarrow I$ are isomorphisms of left and right $U L$-modules, respectively.

Assume that $Y$ is obtained by a free cell attachment $\bigvee_{j \in J} S^{n_{j}} \stackrel{\bigvee \alpha_{j}}{\longrightarrow} X$. Let $\hat{\alpha_{j}}$ denote the adjoint of $\alpha_{j}$. We will show that $H_{*}(\Omega Y ; R)$ can be determined by calculating the homology of the following simple differential graded Lie algebra $(\mathrm{dgL})$

$$
\underline{\mathbf{L}}=\left(L_{X} \amalg \mathbb{L}\left\langle y_{j}\right\rangle_{j \in J}, d\right) \text {, where } d y_{j}=h_{X}\left(\hat{\alpha_{j}}\right) .
$$

In addition to the usual grading, $\underline{\mathbf{L}}$ has a second grading given by letting $L_{X}$ be in degree 0 and letting each $y_{j}$ be in degree 1 . Remarkably, we will show that for free cell attachments one only needs to calculate $H \underline{\mathbf{L}}$ in degrees 0 and 1.

Let $(H \underline{\mathbf{L}})_{i}$ denote the component of $H \underline{\mathbf{L}}$ in degree $i$. For degree reasons, $(H \underline{\mathbf{L}})_{0}$ acts on $(H \underline{\mathbf{L}})_{1}$ by the adjoint action.

Definition 1.4. Define $f$ to be a semi-inert cell attachment if it is a free cell attachment and $(H \underline{\mathbf{L}})_{1}$ is a free $(H \underline{\mathbf{L}})_{0}$-module.

We will see in Section 2 that there is an obvious filtration on $H_{*}(\Omega Y ; R)$. Let $\operatorname{gr}_{*}\left(H_{*}(\Omega Y ; R)\right)$ be the associated graded object. We will show (see Definition 4.2) 
that a free cell attachment is semi-inert if and only if $\operatorname{gr}_{1}\left(H_{*}(\Omega Y ; R)\right)$ is a free $\operatorname{gr}_{0}\left(H_{*}(\Omega Y ; R)\right)$-bimodule.

Theorem 1.5. Let $Y=X \cup_{f}\left(\bigvee_{j \in J} e^{n_{j}+1}\right)$. Assume that $f$ is free.

(i) Then as algebras

$$
\operatorname{gr}\left(H_{*}(\Omega Y ; R)\right) \cong U\left((H \underline{\mathbf{L}})_{0} \ltimes \mathbb{L}\left((H \underline{\mathbf{L}})_{1}\right)\right)
$$

with $(H \underline{\mathbf{L}})_{0} \cong L_{X} /\left[L_{X}^{W}\right]$ as Lie algebras.

(ii) Furthermore if $f$ is semi-inert, then for some $K^{\prime}$,

$$
H_{*}(\Omega Y ; R) \cong U\left(L_{Y}^{X} \amalg \mathbb{L} K^{\prime}\right)
$$

as algebras.

The above result is given more precisely in Theorem 4.3. This theorem is a nearly direct translation of a purely algebraic result given in Theorem 3.12 This algebraic result may have other applications such as the calculation of the mod $p$ Bockstein spectral sequence (BSS) of finite CW-complexes. Scott [Sco02] has shown that for sufficiently large $p$ each term in the mod $p$ BSS of such spaces is a universal enveloping algebra of a dgL.

Corollary 1.6. Free cell attachments are nice in the sense of Hess and Lemaire [HL96].

When $R=\mathbb{Q}$, Milnor and Moore [MM65] proved the fabulous result that the canonical algebra map $U\left(\pi_{*}(\Omega Y) \otimes \mathbb{Q}\right) \rightarrow H_{*}(\Omega Y ; \mathbb{Q})$ is an isomorphism. Scott [Sco03] generalized this result to $R \subset \mathbb{Q}$ for finite CW-complexes when certain primes are invertible in $R$. For an $R$-module $M$, let $F M=M / \operatorname{Torsion}(M)$. Let $P$ denote the primitive elements of $F H_{*}(\Omega Y ; R)$ which are a Lie subalgebra. Scott showed that $U P \stackrel{\cong}{\longrightarrow} F H_{*}(\Omega Y ; R)$ and that $F\left(\pi_{*}(\Omega Y) \otimes R\right)$ injects into $P$. However he showed that in general this injection is not a surjection.

We will give sufficient conditions under which one obtains the desired isomor$\operatorname{phism} U F\left(\pi_{*}(\Omega Y) \otimes R\right) \rightarrow F H_{*}(\Omega Y ; R)$.

In Section 5 we will assume that the Hurewicz map $h_{X}$ has a right inverse. Using this map we will define the set of implicit primes of $Y$. Intuitively, they are the primes $p$ for which $p$-torsion is used in the attaching map $f$. Let

$$
\mathcal{S}=\left\{S^{2 m-1}, \Omega S^{2 m+1} \mid m \geq 1\right\}
$$

Let $\prod \mathcal{S}$ be the collection of spaces homotopy equivalent to a weak product of spaces in $\mathcal{S}$.

Theorem 1.7. Let $Y=X \cup_{f}\left(\bigvee e^{n_{j}+1}\right)$. Assume that $f$ is free, that the Hurewicz map $h_{X}$ has a right inverse, and that the implicit primes are invertible. Then the canonical algebra map

$$
U L_{Y} \rightarrow H_{*}(\Omega Y ; R)
$$

is a surjection. Furthermore if $R \subset \mathbb{Q}$, then (1.1) is an isomorphism and localized at $R, \Omega Y \in \prod \mathcal{S}$. If in addition $f$ is semi-inert, then

$$
L_{Y} \cong H \underline{\mathbf{L}} \cong(H \underline{\mathbf{L}})_{0} \amalg \mathbb{L}\left((H \underline{\mathbf{L}})_{1}\right)
$$

as Lie algebras, and $h_{Y}$ has a right inverse.

Note that the surjection of (1.1) implies that $H_{*}(\Omega Y ; R)$ is generated as an algebra by Hurewicz images. Again, more details are given in Theorem 5.5 . 
Corollary 1.8. If $R \subset \mathbb{Q}$, then the canonical algebra map

$$
U F\left(\pi_{*}(\Omega Y) \otimes R\right) \rightarrow H_{*}(\Omega Y ; R)
$$

is an isomorphism.

Corollary 1.9. If $Z$ is a finite cell complex constructed using only semi-inert cell attachments, then localized away from a finite set of primes, $\Omega Z \in \prod \mathcal{S}$.

It is a long-standing conjecture of Avramov [Avr82] and Félix [FHT84] that if $Z$ has finite LS category, then $L_{Z}$ is either finite dimensional or contains a free Lie subalgebra on two generators. Our final corollary provides further support for this conjecture.

Corollary 1.10. If $R \subset \mathbb{Q}, f$ is a free cell attachment and $\operatorname{dim}(H \underline{\mathbf{L}})_{1}>1$, then $L_{Y}$ contains a free Lie subalgebra on two generators.

We conclude by giving examples of spaces constructed out of semi-inert cell attachments, together with their Hurewicz images. In particular, we give an infinite family of finite CW-complexes and an uncountable family of finite-type CWcomplexes.

Outline of the paper. In Section 2 we will translate the cell attachment problem to a purely algebraic problem using Adams-Hilton models. We then prove our main algebraic results in Section 3 In Section 4 we translate our algebraic results to obtain Theorem 1.5 In Section 5 we prove results about Hurewicz images and homotopy type to obtain Theorem 1.7 Finally in Section 6 we apply our results to study some examples.

\section{Adams-Hilton models}

Let $R=\mathbb{F}_{p}$ with $p>3$ or let $R$ be a subring of $\mathbb{Q}$ containing $\frac{1}{6}$.

A simply-connected space $X$ has an Adams-Hilton model AH56] which we denote by $\mathbf{A}(X) . \mathbf{A}(X)$ is a connected differential graded algebra (dga) which comes with a chain map $\mathbf{A}(X) \rightarrow C_{*}(\Omega X ; R)$ which induces an isomorphism of algebras $H(\mathbf{A}(X)) \stackrel{\cong}{\rightrightarrows} H_{*}(\Omega X ; R)$.

Given a cell attachment $f: W \rightarrow X$ where $W=\bigvee_{j \in J} S^{n_{j}}$ and $f=\bigvee_{j \in J} \alpha_{j}$, let $Y$ be the adjunction space $Y=X \cup_{f}\left(\bigvee_{j \in J} e^{n_{j}+1}\right)$. It is a property of AdamsHilton models that one can choose the following Adams-Hilton model for $Y$ :

$$
\mathbf{A}(Y)=\mathbf{A}(X) \amalg \mathbb{T}\left\langle y_{j}\right\rangle_{j \in J},
$$

where $\mathbb{T}$ denotes the tensor algebra. The differential on $y_{j}$ is determined using the attaching map $\alpha_{j}$.

Filter $\mathbf{A}(Y)$ by the 'length in $y_{k}$ 's' filtration. That is, let $F_{-1} \mathbf{A}(Y)=0$, let $F_{0} \mathbf{A}(Y)=\mathbf{A}(X)$, and for $i \geq 0$, let $F_{i+1} \mathbf{A}(Y)=F_{i} \mathbf{A}(Y)+\sum_{k=0}^{i} F_{k} \mathbf{A}(Y)$. $R\left\{y_{j}\right\}_{j \in J} \cdot F_{i-k} \mathbf{A}(Y)$. This filtration makes $\mathbf{A}(Y)$ a filtered dga.

This filtration induces a first quadrant multiplicative spectral sequence with $E_{p, q}^{0}=\left[F_{p} \mathbf{A}(Y) / F_{p-1} \mathbf{A}(Y)\right]_{p+q}$ which converges from $\operatorname{gr}(\mathbf{A}(Y))$ to $\operatorname{gr}(H \mathbf{A}(Y))$.

Assume that $H_{*}(\Omega X ; R) \cong U L_{X}$ as algebras and that it is $R$-free. Then $\left(E^{1}, d^{1}\right) \cong\left(U L_{X} \amalg U \mathbb{L}\left\langle y_{j}\right\rangle_{j \in J}, d^{\prime}\right)$, where $\mathbb{L}$ denotes the free Lie algebra and $d^{\prime}$ is determined by the induced map $d^{\prime}: R\left\{y_{j}\right\} \stackrel{d}{\rightarrow} Z \mathbf{A}(X) \rightarrow H_{*}(\Omega X ; R) \stackrel{\cong}{\longrightarrow} U L_{X}$. 
It follows from the definition of the differential that $d^{\prime} y_{j}=h_{X}\left(\hat{\alpha}_{j}\right)$, where $\hat{\alpha}_{j}$ is the adjoint of $\alpha_{j}$. Therefore $d^{\prime} y_{j} \in L_{X}$. Thus

$$
\left(E^{1}, d^{1}\right) \cong U \underline{\mathbf{L}}, \text { where } \underline{\mathbf{L}}=\left(L_{X} \amalg \mathbb{L}\left\langle y_{j}\right\rangle, d^{\prime}\right) .
$$

\section{Differential graded algebra extensions}

Let $R=\mathbb{Q}$ or $\mathbb{F}_{p}$ where $p>3$ or let $R$ be a subring of $\mathbb{Q}$ containing $\frac{1}{6}$. All of our $R$-modules are graded and will be assumed to have finite type.

If $R \subset \mathbb{Q}$, then let $P$ be the set of invertible primes in $R$ and let

$$
\tilde{P}=\{p \in \mathbb{Z} \mid p \text { is prime and } p \notin P\} \cup\{0\} .
$$

Notation 3.1. Let $\mathbb{F}_{0}$ denote $\mathbb{Q}$. If $M$ is an $R$-module, then for each $p \in \tilde{P}$ we will denote $M \otimes \mathbb{F}_{p}$ by $\bar{M}$ omitting $p$ from the notation. Similarly if $d$ is a differential we will denote $d \otimes \mathbb{F}_{p}$ by $\bar{d}$.

Let $(A, d)$ be a connected finite-type differential graded algebra (dga) over $R$ which is $R$-free. Let $Z A$ denote the subalgebra of cycles of $A$. Let $V_{1}$ be a connected finite-type free $R$-module and let $d: V_{1} \rightarrow Z A$. Then there is a canonical dga extension $\mathbf{B}=\left(A \amalg \mathbb{T} V_{1}, d\right)$.

Assume that for some Lie algebra $L_{0}$ which is a free $R$-module, $H(A, d) \cong U L_{0}$ as algebras. There is an induced map $d^{\prime}: V_{1} \stackrel{d}{\rightarrow} Z A \rightarrow H A \stackrel{\cong}{\longrightarrow} U L_{0}$. Assume that $L_{0}$ can be chosen such that $d^{\prime} V_{1} \subset L_{0}$.

Taking $d^{\prime} L_{0}=0$, there is a canonical differential graded Lie algebra $(\operatorname{dgL})$

$$
\underline{\mathbf{L}}=\left(L_{0} \amalg \mathbb{L} V_{1}, d^{\prime}\right) .
$$

Then $\underline{\mathbf{L}}$ is a bigraded $\operatorname{dgL}$ where the usual grading is called dimension and a second grading, called degree, is given by taking $L_{0}$ and $V_{1}$ to be in degrees 0 and 1 , respectively. Then the differential $d$ has bidegree $(-1,-1)$.

Notation 3.2. Subscripts of bigraded objects will denote degree, e.g. $M_{0}$ is the component of $M$ in degree 0 .

The following lemma is a well-known fact, and the subsequent lemma is part of lemmas from [Ani89]. We remind the reader that all of our $R$-modules have finite type.

Lemma 3.3. Let $R \subset \mathbb{Q}$. A homomorphism $f: M \rightarrow N$ is an isomorphism if and only if for each $p \in \tilde{P}, f \otimes \mathbb{F}_{p}$ is an isomorphism.

Let $L$ be a connected bigraded dgL. The inclusion $L \hookrightarrow U L$ induces a natural map

$$
\psi: U H L \rightarrow H U L
$$

Lemma 3.4 ([ Ani89, Lemmas 4.1 and 4.3]). Let $R=\mathbb{F}_{p}$ with $p>3$ or let $R \subset \mathbb{Q}$ containing $\frac{1}{6}$. Suppose that $H U L$ is $R$-free in degrees 0 and 1 . Then $H L$ is $R$-free in degrees 0 and 1 and the map $\psi$ in (3.1) is an isomorphism in degrees 0 and 1 .

$\mathbf{B}$ is a filtered dga under the increasing filtration given by $F_{-1} \mathbf{B}=0, F_{0} \mathbf{B}=A$, and for $i \geq 0, F_{i+1} \mathbf{B}=\sum_{j=0}^{i} F_{j} \mathbf{B} \cdot V_{1} \cdot F_{i-j} \mathbf{B}$. Letting

$$
E_{p, q}^{0}(\mathbf{B})=\left[F_{p}(\mathbf{B}) / F_{p-1}(\mathbf{B})\right]_{p+q}
$$


gives a first quadrant spectral sequence of algebras:

$$
\left(E^{0}(\mathbf{B}), d^{0}\right)=\operatorname{gr}(\mathbf{B}) \Longrightarrow E^{\infty}=\operatorname{gr}(H \mathbf{B}) .
$$

It is easy to check that $\left(E^{1}, d^{1}\right) \cong U \underline{\mathbf{L}}$ and hence $E^{2} \cong H U \underline{\mathbf{L}}$. Theorem 3.5 below follows from the main result of Anick's thesis [Ani82, Theorem 3.7]. Anick's theorem holds under either of two hypotheses. We will use only one of these.

Recall that the Hilbert series of an $\mathbb{F}$-module is given by the power series $A(z)=$ $\sum_{n=0}^{\infty}\left(\operatorname{Rank}_{\mathbb{F}} A_{n}\right) z^{n}$. Assuming that $A_{0} \neq 0$, the notation $(A(z))^{-1}$ denotes the power series $1 /(A(z))$.

Theorem 3.5. Let $R=\mathbb{F}$. If the two-sided ideal $\left(d^{\prime} V_{1}\right) \subset U L_{0}$ is a free $U L_{0}$ module, then the above spectral sequence collapses at the $E^{2}$ term. That is, $\operatorname{gr}(H \mathbf{B})$ $\cong H U \underline{\mathbf{L}}$ as algebras. Furthermore the multiplication map

$$
\nu: \mathbb{T}\left(\psi(H \underline{\mathbf{L}})_{1}\right) \otimes(H U \underline{\mathbf{L}})_{0} \rightarrow H U \underline{\mathbf{L}}
$$

is an isomorphism and $(H U \underline{\mathbf{L}})_{0} \cong U L_{0} /\left(d^{\prime} V_{1}\right)$. In addition,

$$
H \mathbf{B}(z)^{-1}=H U \underline{\mathbf{L}}(z)^{-1}=(1+z)(H U \underline{\mathbf{L}})_{0}(z)^{-1}-z\left(U L_{0}\right)(z)^{-1}-V_{1}(z) .
$$

Proof. [Ani82, Theorem 3.7] shows that the spectral sequence collapses as claimed and that the multiplication map $\mathbb{T} W \otimes(H U \underline{\mathbf{L}})_{0} \rightarrow H U \underline{\mathbf{L}}$ is an isomorphism where $W$ is a basis for $(H U \underline{\mathbf{L}})_{1}$ as a right $(H U \underline{\mathbf{L}})_{0}$-module. By Lemma 3.4 and the Poincaré-Birkhoff-Witt Theorem, the homomorphism $\psi(H \underline{\mathbf{L}})_{1} \otimes(H U \underline{\mathbf{L}})_{0} \rightarrow(H U \underline{\mathbf{L}})_{1}$ induced by multiplication in $H U \underline{\mathbf{L}}$ is an isomorphism. So we can let $W=\psi(H \underline{\mathbf{L}})_{1}$.

The remainder of the theorem follows directly from [Ani82, Theorem 3.7].

Corollary 3.6. If $R \subset \mathbb{Q}$ and for each $p \in \tilde{P}$ the two-sided ideal $\left(\bar{d} \bar{V}_{1}\right) \subset U \bar{L}_{0}$ is a free $U \bar{L}_{0}$-module, then $H \mathbf{B}$ is $R$-free if and only if $H U \underline{\mathbf{L}}$ is $R$-free if and only if $L_{0} /\left[d^{\prime} V_{1}\right]$ is $R$-free.

Proof. First $(H U \underline{\mathbf{L}})_{0} \cong U L_{0} /\left(d^{\prime} V_{1}\right) \cong U\left(L_{0} /\left[d^{\prime} V_{1}\right]\right)$. So $(H U \underline{\mathbf{L}})_{0}$ is $R$-free if and only if $L_{0} /\left[d^{\prime} V_{1}\right]$ is $R$-free. Since $U L_{0}$ and $V_{1}$ are $R$-free, the corollary follows from (3.2).

We now prove a version of Theorem 3.5 for subrings of $\mathbb{Q}$.

Theorem 3.7. Let $R \subset \mathbb{Q}$. If $L_{0} /\left[d^{\prime} V_{1}\right]$ is $R$-free and for each $p \in \tilde{P}$ the two-sided ideal $\left(\bar{d} \bar{V}_{1}\right) \subset U \bar{L}_{0}$ is a free $U \bar{L}_{0}$-module, then $H \mathbf{B}$ is $R$-free and the multiplication map

$$
\nu: \mathbb{T}\left(\psi(H \underline{\mathbf{L}})_{1}\right) \otimes(H U \underline{\mathbf{L}})_{0} \rightarrow H U \underline{\mathbf{L}}
$$

is an isomorphism. Also $\operatorname{gr}(H \mathbf{B}) \cong H U \underline{\mathbf{L}}$ as algebras and $(H U \underline{\mathbf{L}})_{0} \cong U L_{0} /\left(d^{\prime} V_{1}\right)$.

Proof. Since $L_{0} /\left[d^{\prime} V_{1}\right]$ is $R$-free, by Corollary 3.6 so are $H U \underline{\mathbf{L}}$ and $H \mathbf{B}$. It follows from the Universal Coefficient Theorem that $\forall p \in \tilde{P}, H \mathbf{B} \otimes \mathbb{F}_{p} \cong H\left(\mathbf{B} \otimes \mathbb{F}_{p}\right)$ and $H U \underline{\mathbf{L}} \otimes \mathbb{F}_{p} \cong H U\left(\underline{\mathbf{L}} \otimes \mathbb{F}_{p}\right)$. In particular $\forall p \in \tilde{P},(H U \underline{\mathbf{L}})_{0} \otimes \mathbb{F}_{p} \cong\left(H U\left(\underline{\mathbf{L}} \otimes \mathbb{F}_{p}\right)\right)_{0}$. Using Lemma 3.4 ,

$$
\psi(H \underline{\mathbf{L}})_{1} \otimes \mathbb{F}_{p} \cong(H \underline{\mathbf{L}})_{1} \otimes \mathbb{F}_{p} \cong H\left(\underline{\mathbf{L}} \otimes \mathbb{F}_{p}\right)_{1} \cong \psi H\left(\underline{\mathbf{L}} \otimes \mathbb{F}_{p}\right)_{1} .
$$

Thus $\forall p \in \tilde{P}$,

$$
\nu \otimes \mathbb{F}_{p}: \mathbb{T}\left(\psi(H \underline{\mathbf{L}})_{1} \otimes \mathbb{F}_{p}\right) \otimes(H U \underline{\mathbf{L}})_{0} \otimes \mathbb{F}_{p} \rightarrow H \mathbf{B} \otimes \mathbb{F}_{p}
$$

corresponds under these isomorphisms to the multiplication map

$$
\mathbb{T}\left(\psi\left(H\left(\underline{\mathbf{L}} \otimes \mathbb{F}_{p}\right)\right)_{1}\right) \otimes\left(H U\left(\underline{\mathbf{L}} \otimes \mathbb{F}_{p}\right)\right)_{0} \rightarrow H\left(\mathbf{B} \otimes \mathbb{F}_{p}\right) .
$$


But this is an isomorphism by Theorem 3.5 Therefore $\nu$ is an isomorphism by Lemma 3.3

The last two isomorphisms also follow from Theorem 3.5.

The next lemma will prove that if the Lie ideal $\left[d^{\prime} V_{1}\right] \subset L_{0}$ is a free Lie algebra, then the hypothesis in Anick's Theorem (Theorem 3.5 holds. That is, $\left(d^{\prime} V_{1}\right)$ is a free $U L_{0}$-module.

Lemma 3.8. Given a dgL $L$ over a field $\mathbb{F}$, denote $U L$ by $A$. Let $J$ be a Lie ideal of $L$ which is a free Lie algebra, $\mathbb{L} W$. Take $I$ to be the two-sided ideal of $A$ generated by $J$. Then the multiplication maps $A \otimes W \rightarrow I$ and $W \otimes A \rightarrow I$ are isomorphisms of left and right $A$-modules, respectively.

Proof. From the short exact sequence of Lie algebras

$$
0 \rightarrow J \rightarrow L \rightarrow L / J \rightarrow 0
$$

we get the short exact of sequence of Hopf algebras

$$
\mathbb{F} \rightarrow U(J) \rightarrow U(L) \rightarrow U(L / J) \rightarrow \mathbb{F}
$$

and so $U L \cong U J \otimes U(L / J)$ as $\mathbb{F}$-modules. Since $J$ is a free Lie algebra $\mathbb{L} W$, $U J \cong \mathbb{T} W$. It is also a basic fact that $U(L / J) \cong U L / I$. Hence we have that

$$
A \cong T W \otimes A / I
$$

as $\mathbb{F}$-modules. Furthermore

$$
A \cong I \oplus A / I
$$

as $\mathbb{F}$-modules.

Let $M(z)$ denote the Hilbert series for the $\mathbb{F}$-module $M$, and to simplify the notation let $B=A / I$. Then from equations (3.3) and (3.4) we have the following (using $(T W)(z)=1 /(1-W(z)))$ :

$$
B(z)=A(z)(1-W(z)), \quad I(z)=A(z)-B(z) .
$$

Combining these we have $I(z)=A(z) W(z)$. That is, $I \cong A \otimes W$ as $\mathbb{F}$-modules.

Let $\mu: A \otimes W \rightarrow I$ be the multiplication map. To show that it is an isomorphism it remains to show that it is either injective or surjective.

We claim that $\mu$ is surjective. Since $I$ is the ideal in $A$ generated by $W$, any $x \in I$ can be written as

$$
x=\sum_{i} a_{i} w_{i} b_{i_{1}} \cdots b_{i_{m_{i}}}, \text { where } a_{i} \in A, w_{i} \in W \text { and } b_{i_{k}} \in L .
$$

Each such expression gives a sequence of numbers $\left\{m_{i}\right\}$. Let $M(x)=\min \left[\max _{i}\left(m_{i}\right)\right]$, where the minimum is taken over all possible ways of writing $x$ as in (3.5). We claim that $M(x)=0$.

Assume that $M(x)=t>0$. Then $x=x^{\prime}+\sum_{i} a_{i} w_{i} b_{i_{1}} \cdots b_{i_{t}}$, where $M\left(x^{\prime}\right)<t$. Now $w_{i} b_{i_{1}}=\left[w_{i}, b_{i_{1}}\right] \pm b_{i_{1}} w_{i}$. Furthermore since $J$ is a Lie ideal, $\left[w_{i}, b_{i_{1}}\right] \in J \cong \mathbb{L} W$, so

$$
\left[w_{i}, b_{i_{1}}\right]=\sum_{j} c_{j}\left[\cdots\left[w_{j_{1}}, w_{j_{2}}\right], \ldots, w_{j_{n_{j}}}\right]=\sum_{k} d_{k} w_{k_{1}} \cdots w_{k_{N_{k}}}=\sum_{l} a_{l} w_{l},
$$

where $a_{l} \in A$ and $w_{l} \in W$. So $x=x^{\prime}+\sum_{i} \sum_{l_{i}} a_{i} a_{l_{i}} w_{l_{i}} b_{i_{2}} \cdots b_{i_{t}}$. But this is of the form in (3.5) and shows that $M(x)<t$, which is a contradiction. 
Therefore for each $x \in I, M(x)=0$ and we can write $x=\sum_{i} a_{i} w_{i}$, where $a_{i} \in A$ and $w_{i} \in W$. Then $x \in \operatorname{im}(\mu)$ and hence $\mu$ is an isomorphism.

Since $A$ is associative, $\mu$ is a map of left $A$-modules.

The second isomorphism follows similarly.

We are now almost ready to prove our main algebraic results. Recall that $\mathbf{B}=$ $\left(A \amalg \mathbb{T} V_{1}, d\right)$, where $d A \subset A$ and $d V_{1} \subset Z A$. Also $H(A, d) \cong U L_{0}$ as algebras and if $d^{\prime}: V_{1} \rightarrow U L_{0}$ is the induced map, then $d^{\prime}\left(V_{1}\right) \subset L_{0}$. Let $\underline{\mathbf{L}}=\left(L_{0} \amalg \mathbb{L} V_{1}, d^{\prime}\right)$ with $d^{\prime} L_{0}=0$.

We introduce the following terminology.

Definition 3.9. If $R$ is a field, say $\mathbf{B}$ is a free dga extension if the Lie ideal $\left[d^{\prime} V_{1}\right] \subset L_{0}$ is a free Lie algebra. If $R \subset \mathbb{Q}$, say $\mathbf{B}$ is a free dga extension if $L_{0} /\left[d^{\prime} V_{1}\right]$ is $R$-free and, using Notation 3.1 for every $p \in \tilde{P}$, the Lie ideal $\left[\bar{d}^{\prime} \bar{V}_{1}\right] \subset \bar{L}_{0}$ is a free Lie algebra.

Definition 3.10. In either of the cases of the previous definition we say $\mathbf{B}$ is a semi-inert dga extension if in addition there is a free $R$-module $K$ such that $(H \underline{\mathbf{L}})_{0} \ltimes \mathbb{L}(H \underline{\mathbf{L}})_{1} \cong(H \underline{\mathbf{L}})_{0} \amalg \mathbb{L} K$. At the end of this section we will give two simpler equivalent conditions (see Lemma 3.13).

Note that it follows from [HL87, Theorem 3.3] and [FT89, Theorem 1] that the semi-inert condition is a generalization of the inert condition.

Recall that there is a map $\psi: U H \underline{\mathbf{L}} \rightarrow H U \underline{\mathbf{L}} . \quad \mathbf{B}$ is a filtered dga under the increasing filtration given by $F_{-1} \mathbf{B}=0, F_{0} \mathbf{B}=A$, and for $i \geq 0, F_{i+1}=$ $\sum_{k=0}^{i} F_{k} \mathbf{B} \cdot V_{1} \cdot F_{i-k} \mathbf{B}$. There is an induced filtration on $H \mathbf{B}$. We prove one last lemma.

Lemma 3.11. There exists a quotient map

$$
f: F_{1} H \mathbf{B} \rightarrow(H U \underline{\mathbf{L}})_{1} .
$$

Given $\bar{w} \in(H \underline{\mathbf{L}})_{1}$ there exists a cycle $w \in F_{1} \mathbf{B}$ such that $f([w])=\bar{w}$.

Proof. By Theorem 3.5 or Theorem 3.7, $(\operatorname{gr}(H \mathbf{B}))_{1} \cong(H U \underline{\mathbf{L}})_{1}$. So there is a quotient map

$$
f: F_{1} H \mathbf{B} \rightarrow(\operatorname{gr}(H \mathbf{B}))_{1} \stackrel{\cong}{\longrightarrow}(H U \underline{\mathbf{L}})_{1} .
$$

By Lemma $3.4(H \underline{\mathbf{L}})_{1} \cong(\underline{\psi} H \underline{\mathbf{L}})_{1} \subset(H U \underline{\mathbf{L}})_{1}$. So for $\bar{w}$ one can choose a representative cycle $w \in Z F_{1} \mathbf{B}$ such that $f([w])=\bar{w}$.

Recall that $R=\mathbb{F}_{p}$ with $p>3$ or $R \subset \mathbb{Q}$ containing $\frac{1}{6}$. Also all of our $R$-modules are connected, $R$-free, and have finite type. Let $(A, d)$ be a dga and let $V_{1}$ be a $R$-module with a map $d: V_{1} \rightarrow A$. Assume that there exists a Lie algebra $L_{0}$ such that $H(A, d) \cong U L_{0}$ as algebras and $d^{\prime} V_{1} \subset L_{0}$, where $d^{\prime}$ is the induced map.

Theorem 3.12. Let $\mathbf{B}=\left(A \amalg \mathbb{T} V_{1}, d\right)$. Assume that $\mathbf{B}$ is a free dga extension in the sense of Definition[3.9. Let $\underline{\mathbf{L}}=\left(L_{0} \amalg \mathbb{L} V_{1}, d^{\prime}\right)$.

(a) Then as algebras

$$
\operatorname{gr}(H \mathbf{B}) \cong U\left((H \underline{\mathbf{L}})_{0} \ltimes \mathbb{L}(H \underline{\mathbf{L}})_{1}\right)
$$

with $(H \underline{\mathbf{L}})_{0} \cong L_{0} /\left[d^{\prime} V_{1}\right]$ as Lie algebras. If $R \subset \mathbb{Q}$, then additionally $(H \underline{\mathbf{L}})_{0} \ltimes$ $\mathbb{L}(H \underline{\mathbf{L}})_{1} \cong \underline{\underline{L}} \underline{H} \underline{\mathbf{L}}$ as Lie algebras. 
(b) Furthermore if $\mathbf{B}$ is semi-inert (that is, there is an R-module $K$ such that $\left.(H \underline{\mathbf{L}})_{0} \ltimes \mathbb{L}(H \underline{\mathbf{L}})_{1} \cong(H \underline{\mathbf{L}})_{0} \amalg \mathbb{L} K\right)$, then as algebras

$$
H \mathbf{B} \cong U\left((H \underline{\mathbf{L}})_{0} \amalg \mathbb{L} K^{\prime}\right)
$$

for some $K^{\prime} \subset F_{1} H \mathbf{B}$ such that $f: K^{\prime} \cong K$, where $f$ is the quotient map in Lemma 3.11

Proof. (a) If $R=\mathbb{F}_{p}$, then by Lemma 3.8, $\left(d^{\prime} V_{1}\right) \subset U L_{0}$ is a free $U L_{0}$-module. If $R \subset \mathbb{Q}$, then by Lemma 3.8 for each $p \in \tilde{P},\left(\bar{d}^{\prime} \bar{V}_{1}\right) \subset U \bar{L}_{0}$ is a free $U \bar{L}_{0}$-module. So we can apply either Theorem 3.5 or Theorem 3.7 to show that $\operatorname{gr}(H \mathbf{B}) \cong H U \underline{\mathbf{L}}$ as algebras and that the multiplication map

$$
\nu: \mathbb{T}\left(\underline{\psi}(H \underline{\mathbf{L}})_{1}\right) \otimes(H U \underline{\mathbf{L}})_{0} \rightarrow H U \underline{\mathbf{L}}
$$

is an isomorphism.

By Lemma $3.4(H U \underline{\mathbf{L}})_{0} \cong U(H \underline{\mathbf{L}})_{0}$ and $\psi(H \underline{\mathbf{L}})_{1} \cong(H \underline{\mathbf{L}})_{1}$. By the definition of homology $(H \underline{\mathbf{L}})_{0} \cong L_{0} /\left[d^{\prime} V_{1}\right]$.

Let $N=\underline{\psi}(H \underline{\mathbf{L}})$. Then $N_{0}$ acts on $N_{1}$ so we can define

$$
L^{\prime}=N_{0} \ltimes \mathbb{L} N_{1} .
$$

This semi-direct product can be thought of as the free Lie algebra generated by $N_{0}$ and $N_{1}$ modulo the relations generated by the action of $N_{0}$ on $N_{1}$. So there is an induced Lie algebra map $u: L^{\prime} \rightarrow N$ and an induced algebra map $\tilde{u}: U L^{\prime} \stackrel{U u}{\longrightarrow}$ $U N \rightarrow H U \underline{\mathbf{L}}$.

Recall that as $R$-modules, $L^{\prime} \cong N_{0} \times \mathbb{L} N_{1}$. The Poincaré-Birkhoff-Witt Theorem shows that the multiplication map

$$
\phi: \mathbb{T} N_{1} \otimes(H U \underline{\mathbf{L}})_{0} \stackrel{\cong}{\longrightarrow} U \mathbb{L} N_{1} \otimes U N_{0} \rightarrow U L^{\prime}
$$

is an isomorphism. Since $\tilde{u}$ is an algebra map, $\nu=\tilde{u} \circ \phi$. Thus $\tilde{u}$ is an isomorphism. Therefore $H U \underline{\mathbf{L}} \cong U L^{\prime}$ as algebras and hence $\operatorname{gr}(H \mathbf{B}) \cong U L^{\prime}$. If $R=\mathbb{F}_{p}$, then this finishes (a).

If $R \subset \mathbb{Q}$, then we will show that $u: L^{\prime} \rightarrow N$ is an isomorphism. Let $\iota: N \hookrightarrow$ $H U \underline{\mathbf{L}}$ be the inclusion. Since the composition $L^{\prime} \stackrel{u}{\rightarrow} N \stackrel{\iota}{\rightarrow} H U \underline{\mathbf{L}} \stackrel{\cong}{\rightarrow} U L^{\prime}$ is the canonical inclusion $L^{\prime} \hookrightarrow U L^{\prime}, u$ is injective. The inclusion $L^{\prime} \rightarrow U L^{\prime}$ splits as $R$-modules; so as $R$-modules $N \cong L^{\prime} \oplus N / L^{\prime}$. Since $L^{\prime}$ and $N$ are $R$-free, so is $N / L^{\prime}$

Recall that the composition $\iota \circ u$ induces the isomorphism $\tilde{u}: U L^{\prime} \stackrel{U u}{\longrightarrow} U N \rightarrow$ $H U \underline{\mathbf{L}}$. Tensor these maps with $\mathbb{Q}$ to get the commutative diagram:

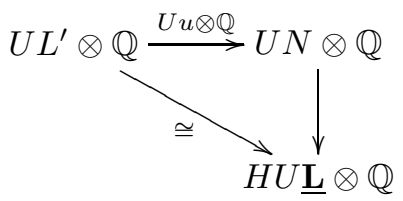

It is a classical result that the natural map

$$
\underline{\psi}_{\mathbb{Q}}: U H(\underline{\mathbf{L}} \otimes \mathbb{Q}) \stackrel{\cong}{\rightrightarrows} H U(\underline{\mathbf{L}} \otimes \mathbb{Q})
$$

is an isomorphism. Note that

$$
N \otimes \mathbb{Q}=(\underline{\psi} H \underline{\mathbf{L}}) \otimes \mathbb{Q} \cong \underline{\psi}_{\mathbb{Q}} H(\underline{\mathbf{L}} \otimes \mathbb{Q}) \cong H(\underline{\mathbf{L}} \otimes \mathbb{Q})
$$


and $H U \underline{\mathbf{L}} \otimes \mathbb{Q} \cong H U(\underline{\mathbf{L}} \otimes \mathbb{Q})$. Under these isomorphisms the vertical map in (3.7) corresponds to the isomorphism in (3.8).

Therefore $U u \otimes \mathbb{Q}$ is an isomorphism and hence $u \otimes \mathbb{Q}$ is surjective. As a result coker $u=N / L^{\prime}$ is a torsion $R$-module. But we have already shown that $N / L^{\prime}$ is $R$-free. Thus $N / L^{\prime}=0$ and $N \cong L^{\prime}$. Hence $H U \underline{\mathbf{L}} \cong U N$.

(b) Recall that $N_{0}$ acts on $N_{1}=(\underline{\psi} H \underline{\mathbf{L}})_{1} \cong(H \underline{\mathbf{L}})_{1}$ via the adjoint action. Assume that $\mathbf{B}$ is semi-inert. That is, there exists $\left\{\bar{w}_{i}\right\} \subset N_{1}$ such that $L^{\prime} \cong$ $N_{0} \amalg \mathbb{L} K$, where $K=R\left\{\bar{w}_{i}\right\}$. Recall from (a) that $H U \underline{\mathbf{L}} \cong \operatorname{gr}(H \mathbf{B})$ and that the inclusions $N_{0} \subset H U \underline{\mathbf{L}}$ and $\bar{w}_{i} \in H U \underline{\mathbf{L}}$ induce a Lie algebra map

$$
u: L^{\prime} \rightarrow \operatorname{gr}(H \mathbf{B}) .
$$

By Lemma 3.11, there exists $w_{i} \in F_{1} \mathbf{B}$ such that $f\left(\left[w_{i}\right]\right)=\bar{w}_{i}$, where $f$ is the map in (3.6). Let $K^{\prime}=R\left\{\left[w_{i}\right]\right\} \subset F_{1} H \mathbf{B}$, and let $L^{\prime \prime}=N_{0} \amalg \mathbb{L} K^{\prime}$. Then $f: K^{\prime} \cong K$ and $f$ induces an isomorphism $L^{\prime \prime} \cong L^{\prime}$.

By part (a), $N_{0} \subset(\text { gr } H \mathbf{B})_{0}$. Since $F_{-1} H \mathbf{B}=0,(\text { gr } H \mathbf{B})_{0} \hookrightarrow H \mathbf{B}$, so $N_{0} \hookrightarrow$ $H \mathbf{B}$. Since $N_{0}, K^{\prime} \hookrightarrow H \mathbf{B}$, there are induced maps

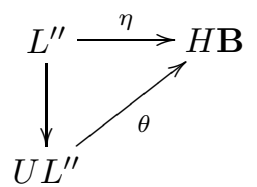

where $\eta$ is a Lie algebra map and $\theta$ is an algebra map.

Grade $L^{\prime \prime}$ by letting $N_{0}$ be in degree 0 and $K^{\prime}$ be in degree 1 . This also filters $L^{\prime \prime}$. Then $\eta$ is a map of filtered objects.

From this we get the following commutative diagram:

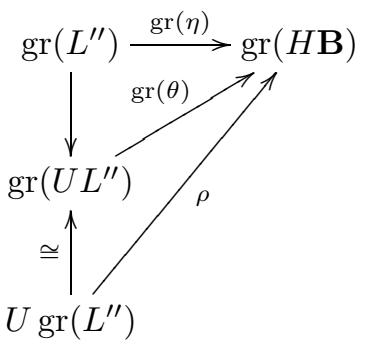

Now $\operatorname{gr}\left(L^{\prime \prime}\right) \cong L^{\prime \prime} \cong L^{\prime}$ and $\operatorname{gr}(\eta)$ corresponds to $u$ under this isomorphism. So $\rho$ corresponds to $\tilde{u}$, which is an isomorphism. Thus $\operatorname{gr}(\theta)$ is an isomorphism, and hence $\theta$ is an isomorphism. Therefore $H \mathbf{B} \cong U L^{\prime \prime}$, which finishes the proof.

As promised we now give two simpler equivalent conditions for semi-inertness.

Lemma 3.13. Let $\mathbf{B}$ be a free dga extension (in the sense of Definition 3.9). Then the following conditions are equivalent:

(a) $(H \underline{\mathbf{L}})_{0} \ltimes \mathbb{L}(H \underline{\mathbf{L}})_{1} \cong(H \underline{\mathbf{L}})_{0} \amalg \mathbb{L} K$ for some free $R$-module $K \subset(H \underline{\mathbf{L}})_{1}$,

(b) $(H \underline{\mathbf{L}})_{1}$ is a free $(H \underline{\mathbf{L}})_{0}-$ module, and

(c) $\operatorname{gr}_{1}(H \mathbf{B})$ is a free $\operatorname{gr}_{0}(H \mathbf{B})$-bimodule.

Proof. (b) $\Longrightarrow$ (a) Let $K$ be a basis for $(H \underline{\mathbf{L}})_{1}$ as a free $(H \underline{\mathbf{L}})_{0}$-module. Then $(H \underline{\mathbf{L}})_{0} \ltimes \mathbb{L}(H \underline{\mathbf{L}})_{1} \cong(H \underline{\mathbf{L}})_{0} \amalg \mathbb{L} K$. 
(a) $\Longrightarrow$ (c) Since $\mathbf{B}$ is a free dga extension, by Theorem $3.12(a), g_{*}(H \mathbf{B}) \cong$ $U\left((H \underline{\mathbf{L}})_{0} \ltimes \mathbb{L}(H \underline{\mathbf{L}})_{1}\right)$. So by (a),

$$
\operatorname{gr}_{*}(H \mathbf{B}) \cong U\left((H \underline{\mathbf{L}})_{0} \amalg \mathbb{L} K\right) \cong \operatorname{gr}_{0}(H \mathbf{B}) \amalg \mathbb{T} K,
$$

for some free $R$-module $K \subset(H \underline{\mathbf{L}})_{1}$. Therefore

$$
\operatorname{gr}_{1}(H \mathbf{B}) \cong\left[\operatorname{gr}_{0}(H \mathbf{B}) \amalg \mathbb{T} K\right]_{1} \cong \operatorname{gr}_{0}(H \mathbf{B}) \otimes K \otimes \operatorname{gr}_{0}(H \mathbf{B}) .
$$

(c) $\Longrightarrow$ (b) Let $L^{\prime}=(H \underline{\mathbf{L}})_{0} \ltimes \mathbb{L}(H \underline{\mathbf{L}})_{1}$. Then by Theorem $3.12(\mathrm{a}), \operatorname{gr}_{*}(H \mathbf{B}) \cong$ $U L^{\prime}$ and $\operatorname{gr}_{1}(H \mathbf{B}) \cong\left(U L^{\prime}\right)_{1}$. By $(\mathrm{c}),\left(U L^{\prime}\right)_{1}$ is a free $\left(U L^{\prime}\right)_{0}$-bimodule. Then we claim that it follows that $N_{1}$ is a free $N_{0}$-module. Indeed, if there is a nontrivial degree one relation in $L^{\prime}$, then there is a corresponding non-trivial degree one relation in $U L^{\prime}$.

\section{Cell attachments}

Let $R=\mathbb{F}_{p}$ with $p>3$ or $R \subset \mathbb{Q}$ containing $\frac{1}{6}$. Let $X$ be a finite-type simply-connected CW-complex such that $H_{*}(\Omega X ; R)$ is torsion-free and as algebras $H_{*}(\Omega X ; R) \cong U L_{X}$, where $L_{X}$ is the Lie algebra of Hurewicz images. Let $W=\bigvee_{j \in J} S^{n_{j}}$ be a finite-type wedge of spheres and let $f: W \rightarrow X$. Let $Y=X \cup_{f}\left(\bigvee_{j \in J} e^{n_{j}+1}\right)$. Using the Adams-Hilton models of Section 2 we defined a Lie algebra $\underline{\mathbf{L}}=\left(L_{X} \amalg \mathbb{L}\left\langle y_{j}\right\rangle, d^{\prime}\right)$. Mirroring Definitions 3.9 and 3.10, we introduce the following terminology.

Definition 4.1. If $R$ is a field call $f$ a free cell attachment if the Lie ideal $\left[L_{X}^{W}\right] \subset$ $L_{X}$ is a free Lie algebra. If $R \subset \mathbb{Q}$ call $f$ a free cell attachment if $L_{X} /\left[L_{X}^{W}\right]$ is $R$-free and for every $p \in \tilde{P}$, the Lie ideal $\left[\bar{L}_{X}^{W}\right] \subset \bar{L}_{X}$ is a free Lie algebra.

Definition 4.2. In either of the cases of the previous definition say that $f$ is a semi-inert cell attachment if in addition one of the following three equivalent (by Lemma 3.13 conditions is satisfied:

(a) $\operatorname{gr}_{1}(H \mathbf{A}(Y))$ is a free $\operatorname{gr}_{0}(H \mathbf{A}(Y))$-bimodule,

(b) $(H \underline{\mathbf{L}})_{1}$ is a free $(H \underline{\mathbf{L}})_{0}$-module, or

(c) there is a free $R$-module $K$ such that

$$
(H \underline{\mathbf{L}})_{0} \ltimes \mathbb{L}(H \underline{\mathbf{L}})_{1} \cong(H \underline{\mathbf{L}})_{0} \amalg \mathbb{L} K .
$$

Theorem 3.12 gives most of the following topological result directly.

Theorem 4.3. Let $Y=X \cup_{f}\left(\bigvee_{j \in J} e^{n_{j}+1}\right)$ and let $\underline{\mathbf{L}}=\left(L_{X} \amalg \mathbb{L}\left\langle y_{j}\right\rangle, d^{\prime}\right)$. Assume that $f$ is free.

(a) Then $H_{*}(\Omega Y ; R)$ and $\operatorname{gr}\left(H_{*}(\Omega Y ; R)\right)$ are $R$-free and as algebras

$$
\operatorname{gr}\left(H_{*}(\Omega Y ; R)\right) \cong U\left(L_{Y}^{X} \ltimes \mathbb{L}(H \underline{\mathbf{L}})_{1}\right)
$$

with $L_{Y}^{X} \cong L_{X} /\left[L_{X}^{W}\right]$ as Lie algebras.

(b) Furthermore if $f$ is semi-inert, then as algebras

$$
H_{*}(\Omega Y ; R) \cong U\left(L_{Y}^{X} \amalg \mathbb{L} K^{\prime}\right)
$$

for some $K^{\prime} \subset F_{1} H_{*}(\Omega Y ; R)$.

Proof. It remains to show that $(H \underline{\mathbf{L}})_{0} \cong L_{Y}^{X}$. By Theorem 3.12 we have the algebra isomorphism

$$
\operatorname{gr}(H \mathbf{A}(Y)) \cong U\left((H \underline{\mathbf{L}})_{0} \ltimes \mathbb{L}(H \underline{\mathbf{L}})_{1}\right)
$$


with $(H \underline{\mathbf{L}})_{0} \cong L_{X} /\left[L_{X}^{W}\right]$. Therefore

$$
F_{0} H \mathbf{A}(Y) \cong(\operatorname{gr}(H \mathbf{A}(Y)))_{0} \cong U(H \underline{\mathbf{L}})_{0} \cong U\left(L_{X} /\left[L_{X}^{W}\right]\right)
$$

The inclusion $i: \mathbf{A}(X) \stackrel{\cong}{\rightrightarrows} F_{0} \mathbf{A}(Y)$ induces a map $H(i): H \mathbf{A}(X) \rightarrow F_{0} H \mathbf{A}(Y)$. Now under the isomorphism (4.1) and $U L_{X} \stackrel{\cong}{\rightarrow} H \mathbf{A}(X)$ the map $H(i)$ corresponds to a map $U L_{X} \rightarrow U\left(L_{X} /\left[L_{X}^{W}\right]\right)$, where $U\left(L_{X} /\left[L_{X}^{W}\right]\right) \subset U L_{Y}$. It is easy to check that this is the canonical map. In other words $L_{Y}^{X} \cong L_{X} /\left[L_{X}^{W}\right]$. Therefore $(H \underline{\mathbf{L}})_{0} \cong$ $L_{Y}^{X}$.

Corollary 1.6 follows from Theorem 4.3

Proof of Corollary 1.6. The cell attachment $f$ is nice in the sense of Hess and Lemaire [HL96] if and only if $U L_{X} /\left(L_{X}^{W}\right)$ injects in $H_{*}(\Omega Y ; R)$. Recall the standard fact that $U L_{X} /\left(L_{X}^{W}\right) \cong U\left(L_{X} /\left[L_{X}^{W}\right]\right)$. By Theorem 4.3 .

$$
U\left(L_{X} /\left[L_{x}^{W}\right]\right) \cong U L_{Y}^{X} \cong \operatorname{gr}_{0}\left(H_{*}(\Omega Y ; R)\right)
$$

which injects in $H_{*}(\Omega Y ; R)$.

\section{Hurewicz IMAGES}

Let $R=\mathbb{F}_{p}$ with $p>3$ or $R \subset \mathbb{Q}$ be a subring containing $\frac{1}{6}$.

Recall that we have a homotopy cofibration $W \stackrel{f}{\rightarrow} X \rightarrow Y$, where $W=\bigvee_{j \in J} S^{n_{j}}$ is a finite-type wedge of spheres, $f=\bigvee_{j \in J} \alpha_{j}, H_{*}(\Omega X ; R)$ is torsion-free, and as algebras $H_{*}(\Omega X ; R) \cong U L_{X}$. For each $j \in J$, let $\hat{\alpha}: S^{n_{j}-1} \rightarrow \Omega X$ denote the adjoint of $\alpha$.

Assume that $f$ is free. That is, the Lie ideal $\left[L_{X}^{W}\right]$ is a free Lie algebra.

Recall that $h_{X}: \pi_{*}(\Omega X) \otimes R \rightarrow L_{X} \subset H_{*}(\Omega X ; R)$ is a Lie algebra map. In order to identify Hurewicz images in $H_{*}(\Omega Y ; R)$ we will need to be able to construct maps from information about the loop space homology. In particular, we will need to assume that there exists a Lie algebra map $\sigma_{X}: L_{X} \rightarrow \pi_{*}(\Omega X) \otimes R$ such that $h_{X} \circ \sigma_{X}=i d_{L_{X}}$. This map exists if $R=\mathbb{Q}$ or if $X$ is a wedge of spheres. We will give a sufficient condition for the existence of this map later in this section.

If $R$ is a subring of $\mathbb{Q}$ with invertible primes $P \supset\{2,3\}$, then we may need to exclude those primes $p$ for which an attaching map $\alpha_{j} \in \pi_{*}(X)$ includes a term with $p$-torsion. Following Anick Ani89 we define the set of implicit primes below.

Definition 5.1. By the Milnor-Moore theorem [MM65, $h_{X}, \sigma_{X}$ are rational isomorphisms, so $\operatorname{im}\left(\sigma_{X} \circ h_{X}-i d\right)$ is a torsion element of $\pi_{*}(\Omega X) \otimes R$. Let $\gamma_{j}=$ $\sigma_{X} h_{X}\left(\hat{\alpha_{j}}\right)-\hat{\alpha}_{j}$, where $\hat{\alpha}_{j}: S^{n_{j}-1} \rightarrow \Omega X$ is the adjoint of $\alpha_{j}$. Then $t_{j} \gamma_{j}=0 \in$ $\pi_{*}(\Omega X) \otimes R$ for some $t_{j}>0$. Let $t_{j}$ be the smallest such integer. Define $P_{Y}$, the set of implicit primes of $Y$, as follows. A prime $p$ is in $P_{Y}$ if and only if $p \in P$ or $p \mid t_{j}$ for some $j \in J$.

One can verify that the implicit primes have the following properties.

Lemma 5.2. (a) Let $\left\{x_{i}\right\}$ be a set of Lie algebra generators for $L_{X}$ and let $\beta_{i}=$ $\sigma_{X} x_{i}$. If all of the attaching maps are R-linear combinations of iterated Whitehead products of the maps $\beta_{i}$, then $P_{Y}=P$.

(b) If $P=\{2,3\}$ and $n=\operatorname{dim}(Y)$, then the implicit primes are bounded by $\max (3, n / 2)$. 
By replacing $R$ with $R^{\prime}=\mathbb{Z}\left[P_{Y}{ }^{-1}\right]$ if necessary, we may assume that the implicit primes are invertible. This implies that for all $j \in J, \sigma_{X} h_{X} \hat{\alpha}_{j}=\hat{\alpha}_{j}$.

Remark 5.3. If $R=\mathbb{F}_{p}$, then we will also need that for all $j \in J, \sigma_{X} h_{X} \hat{\alpha}_{j}=\hat{\alpha}_{j}$. So that we can state the cases $R=\mathbb{F}_{p}$ and $R \subset \mathbb{Q}$ together, when $R=\mathbb{F}_{p}$ and we say the implicit primes are invertible, we mean that for all $j \in J, \sigma_{X} h_{X} \hat{\alpha}_{j}=\hat{\alpha}_{j}$.

We now consider both cases $R=\mathbb{F}_{p}$ or $R \subset \mathbb{Q}$. Recall that in Section 2 we defined the differential graded Lie algebra

$$
\underline{\mathbf{L}}=\left(L_{X} \amalg \mathbb{L}\left\langle y_{j}\right\rangle_{j \in J}, d^{\prime}\right), \quad \text { where } d^{\prime} y_{j}=h_{X} \hat{\alpha}_{j} .
$$

By Theorem 4.3. $H_{*}(\Omega Y ; R)$ is torsion-free and $\operatorname{gr}\left(H_{*}(\Omega Y ; R)\right) \cong U\left(L_{Y}^{X} \ltimes \mathbb{L}(H \underline{\mathbf{L}})_{1}\right)$ as algebras. From this we want to show that $H_{*}(\Omega Y ; R) \cong U L_{Y}$ as algebras.

This situation closely resembles that of torsion-free spherical two-cones, and we will generalize Anick's proof for that situation [Ani89].

The proof of the following is the same as the proof of [Ani89, Claim 4.7], so we will only sketch it here. See [Bub03. Chapter 7] for more details.

Proposition 5.4. Let $W \rightarrow X \rightarrow Y$ and $\underline{\mathbf{L}}$ be as above with $\sigma_{X} h_{X} \hat{\alpha}_{j}=\hat{\alpha_{j}}, \forall j$. Then there exists an injection of $L_{Y}^{X}$-modules

$$
u_{1}:(H \underline{\mathbf{L}})_{1} \hookrightarrow\left(\operatorname{gr}\left(L_{Y}\right)\right)_{1} .
$$

Proof sketch. Let $\bar{w}_{i} \in(H \underline{\mathbf{L}})_{1}$ be a homology class in dimension $m+1$. It can be represented by a cycle $\gamma_{i} \in \underline{\mathbf{L}}$ in degree 1 . Using the Jacobi identity one can write

$$
\gamma_{i}=\sum_{k=1}^{s} c_{k} u_{k} \text { where } c_{k} \in R \text { and } u_{k}=\left[\cdots\left[y_{j_{k}}, x_{k_{1}}\right], \ldots, x_{k_{n_{k}}}\right],
$$

with $\left[x_{k_{i}}\right] \in L_{X}$.

The sphere $S^{m}$ has an Adams-Hilton model $(\mathbb{T}\langle a\rangle, 0)$ which can be extended to $(\mathbb{T}\langle a, b\rangle, d)$, where $d b=a$ which is an Adams-Hilton model for the disk $D^{m+1}$. Using properties of Adams-Hilton models, one can construct maps $g_{k}:\left(D^{m+1}, S^{m}\right) \rightarrow$ $(Y, X)$ for $1 \leq k \leq s$ such that $\mathbf{A}\left(g_{k}\right)(b)=c_{k} u_{k}$.

Let

$$
g^{\prime}=g_{1} \vee \ldots \vee g_{s}:\left(\bigvee_{k=1}^{s} D^{m+1}, \bigvee_{k=1}^{s} S^{m}\right) \rightarrow(Y, X)
$$

Using the fact that $\sigma_{X} h_{X} \hat{\alpha}_{j}=\hat{\alpha_{j}}$, one can show that $g^{\prime} \mid \bigvee_{k} S^{m}$ is contractible in $X$. As a result, $g^{\prime}$ can be extended to a map $g: S^{m+1} \rightarrow Y$ whose Hurewicz image modulo lower filtration is $\bar{w}_{i}$.

From this we will prove our final main result. Recall that $R=\mathbb{F}_{p}$ with $p>3$ or $R \subset \mathbb{Q}$ containing $\frac{1}{6}$.

Theorem 5.5. Let $Y=X \cup_{f}\left(\bigvee e^{n_{j}+1}\right)$ with $f=\bigvee \alpha_{j}$ satisfying the hypotheses of Theorem 4.3. In addition assume that $h_{X}$ has a right inverse $\sigma_{X}$ and that the implicit primes are invertible. Then:

(a) the canonical algebra map

$$
U L_{Y} \rightarrow H_{*}(\Omega Y ; R)
$$

is a surjection.

(b) If $R \subset \mathbb{Q}$, then (5.1) is an isomorphism, $\operatorname{gr}\left(L_{Y}\right) \cong L_{Y}^{X} \ltimes \mathbb{L}\left((H \underline{\mathbf{L}})_{1}\right)$, and localized at $R, \Omega Y \in \prod \mathcal{S}$. 
(c) If $R \subset \mathbb{Q}$ and $f$ is semi-inert, then:

(i) there exists $\hat{K} \subset F_{1} L_{Y}$ such that $L_{Y} \cong L_{Y}^{X} \amalg \mathbb{L} \hat{K}$ as Lie algebras,

(ii) $L_{Y} \cong H \underline{\mathbf{L}}$ as Lie algebras, and

(iii) $h_{Y}$ has a right inverse $\sigma_{Y}$.

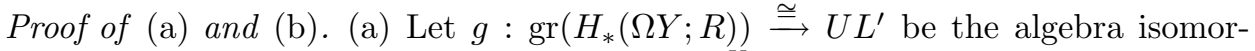
phism given by Theorem 4.3 (a), where $L^{\prime}=L_{Y}^{X} \ltimes \mathbb{L} K^{\prime}$ with $K^{\prime}=(H \underline{\mathbf{L}})_{1}$. Note that $L_{0}^{\prime}=L_{Y}^{X}$ and that $L_{1}^{\prime}=K^{\prime}$.

We have an injection of Lie algebras

$$
u_{0}: L_{Y}^{X} \hookrightarrow F_{0} L_{Y} \stackrel{\cong}{\longrightarrow}\left(\operatorname{gr}\left(L_{Y}\right)\right)_{0} .
$$

Since the implicit primes are invertible, we have that for all $j, h_{X} \sigma_{X} \hat{\alpha}_{j}=\hat{\alpha}_{j}$. So by Proposition [5.4 we get an injection of $L_{Y}^{X}$-modules $u_{1}: K^{\prime} \hookrightarrow\left(\operatorname{gr}\left(L_{Y}\right)\right)_{1}$. Hence for $x \in L_{Y}^{X}$ and $y \in K^{\prime}, u_{1}([y, x])=\left[u_{1}(y), u_{0}(x)\right]$. Thus $u_{0}$ and $u_{1}$ can be extended to a Lie algebra map $u: L^{\prime} \rightarrow \operatorname{gr}\left(L_{Y}\right)$.

The inclusion $L_{Y} \hookrightarrow H_{*}(\Omega Y ; R)$ induces a map between the corresponding graded objects, $\chi: \operatorname{gr}\left(L_{Y}\right) \rightarrow \operatorname{gr}\left(H_{*}(\Omega Y ; R)\right)$.

We claim that for $j=0$ and $1, g \circ \chi \circ u_{j}$ is the ordinary inclusion of $L_{j}^{\prime}$ in $U L^{\prime}$. For $j=0$, under the isomorphisms $\operatorname{gr}_{0} L_{Y} \cong F_{0} L_{Y}$ and $\operatorname{gr}_{0} H_{*}(\Omega Y ; R) \cong F_{0} H_{*}(\Omega Y ; R)$, $g \chi u_{0}$ corresponds to the map $L_{Y}^{X} \hookrightarrow F_{0} L_{Y} \hookrightarrow F_{0} H_{*}(\Omega Y ; R) \stackrel{\cong}{\longrightarrow} U L_{Y}^{X}$. For $j=1$, under the isomorphism $U L^{\prime} \cong H U \underline{\mathbf{L}}, g \chi u_{1}$ corresponds to the inclusion $K^{\prime}=$ $(H \underline{\mathbf{L}})_{1} \hookrightarrow(H U \underline{\mathbf{L}})_{1}$. It follows that $g \circ \chi \circ u$ is the standard inclusion $L^{\prime} \hookrightarrow U L^{\prime}$. Since $g \circ \chi \circ u$ is an injection, so is $u$.

The canonical map $U \operatorname{gr}\left(L_{Y}\right) \stackrel{\cong}{\longrightarrow} \operatorname{gr}\left(U L_{Y}\right)$ is an algebra isomorphism. Now $u$ and $\chi$ induce the maps $U u$ and $\bar{\chi}$ in the following diagram:

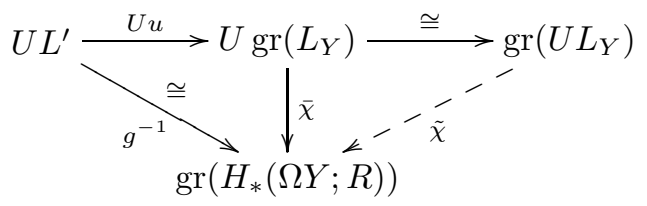

Since we showed that $g \chi u$ is the ordinary inclusion $L^{\prime} \hookrightarrow U L^{\prime}$, the diagram commutes. Since $g^{-1}$ is surjective, the induced map $\tilde{\chi}$ is surjective. Since $\tilde{\chi}$ is the associated graded map to the canonical map $U L_{Y} \rightarrow H_{*}(\Omega Y ; R)$ and the filtrations are bicomplete, the associated ungraded map is also surjective. So the canonical map $U L_{Y} \rightarrow H_{*}(\Omega Y ; R)$ is surjective which finishes the proof of (i).

(b) In the case where $R \subset \mathbb{Q}$, we can tensor with $\mathbb{Q}$ and make use of results from rational homotopy theory.

Recall that $\operatorname{gr}\left(H_{*}(\Omega Y ; R)\right) \cong U L^{\prime}$ and that we constructed a Lie algebra map $u: L^{\prime} \rightarrow \operatorname{gr}\left(L_{Y}\right)$ and showed that it is an injection. We claim that for $R \subset \mathbb{Q}, u$ is an isomorphism. $H_{*}(\Omega Y ; R)$ and $\operatorname{gr}\left(H_{*}(\Omega Y ; R)\right)$ have the same Hilbert series. Also since $H_{*}(\Omega Y ; R)$ is torsion-free, it has the same Hilbert series as $H_{*}(\Omega Y ; \mathbb{Q})$. Let $S$ be the image of $h_{Y} \otimes \mathbb{Q}$. Then $S, L_{Y}$ and $\operatorname{gr}\left(L_{Y}\right)$ have the same Hilbert series. By the Milnor-Moore Theorem [MM65], $H_{*}(\Omega Y ; \mathbb{Q}) \cong U S$. So by the Poincaré-Birkhoff-Witt Theorem, $S$ has the same Hilbert series as $L^{\prime}$, and hence $\operatorname{gr}\left(L_{Y}\right) \cong L^{\prime}$ as $R$-modules. Since $u: L^{\prime} \rightarrow \operatorname{gr}\left(L_{Y}\right)$ is an injection it follows that it is an isomorphism.

Using diagram (5.3) we get that (5.1) is an isomorphism. 
The map $u$ gives the desired Lie algebra isomorphism $\operatorname{gr}\left(L_{Y}\right) \cong L^{\prime}=L_{Y}^{X} \ltimes$ $\mathbb{L}(H \underline{\mathbf{L}})_{1}$.

By the Hilton-Serre-Baues Theorem [Bau81, Lemma V.3.10], Ani89, Lemma 3.1], that (5.1) is an isomorphism is equivalent to the statement that localized at $R, \Omega Y \in \prod \mathcal{S}$.

Before we prove (c) we strengthen the result in (b) in the semi-inert case.

Lemma 5.6. Let $R \subset \mathbb{Q}$. Assume $Y$ is a space satisfying the hypotheses of Theorem [5.5. If furthermore $f$ is semi-inert, then there exists $\hat{K} \subset F_{1} L_{Y}$ such that $L_{Y} \cong L_{Y}^{X} \amalg \mathbb{L} \hat{K}$ as Lie algebras.

Proof. Assume that $f$ is semi-inert. Recall the situation from Theorem 4.3(b). We have that $\operatorname{gr}\left(H_{*}(\Omega Y ; R)\right) \cong U L^{\prime}$, where $L^{\prime} \cong L_{Y}^{X} \amalg \mathbb{L} K^{\prime}$, where $K^{\prime} \cong R\left\{\bar{w}_{i}\right\} \subset$ $\left(\operatorname{gr}\left(H_{*}(\Omega Y ; R)\right)\right)_{1}$. For each $\bar{w}_{i}$ let $\left[w_{i}\right]$ be an inverse image under the quotient map $H_{*}(\Omega Y ; R) \rightarrow \operatorname{gr}\left(H_{*}(\Omega Y ; R)\right)$. Let $K^{\prime \prime}=\left\{\left[w_{i}\right]\right\}$. By Theorem 4.3(b) as algebras $H_{*}(\Omega Y ; R) \cong U L^{\prime \prime}$, where $L^{\prime \prime}=L_{Y}^{X} \amalg \mathbb{L} K^{\prime \prime}$ (see Theorem $\left.3.12(\mathrm{~b})\right)$. Since $K^{\prime \prime} \cong$ $K^{\prime}$, there is an induced Lie algebra isomorphism $L^{\prime \prime} \cong L^{\prime} \cdot \operatorname{So} \operatorname{gr}\left(H_{*}(\Omega Y ; R)\right) \cong$ $H_{*}(\Omega Y ; R)$ as algebras.

Recall that $K^{\prime}=(H \underline{\mathbf{L}})_{1}$, so by Proposition 5.4 there exists $\hat{K} \subset F_{1} L_{Y}$ such that $f: \hat{K} \stackrel{\cong}{\longrightarrow} K^{\prime}$, where $f$ is the quotient map from (3.6). Let

$$
\hat{L}=L_{Y}^{X} \amalg \mathbb{L} \hat{K} \text {. }
$$

So $f: \hat{K} \stackrel{\cong}{\longrightarrow} K^{\prime}$ induces a Lie algebra isomorphism $\hat{L} \cong L^{\prime}$. This in turn induces the algebra isomorphism $U \hat{L} \stackrel{\cong}{\cong} U L^{\prime} \cong H_{*}(\Omega Y ; R)$.

Since $U \hat{L} \cong H_{*}(\Omega Y ; R)$ as algebras, there is an injection $L_{Y} \hookrightarrow U \hat{L}$. Also, since $\hat{K} \subset L_{Y}$, there is a canonical Lie algebra map $v: \hat{L} \rightarrow L_{Y}$. These fit into the following commutative diagram:

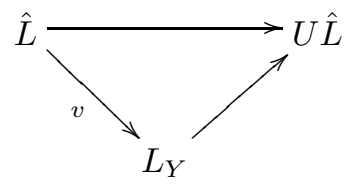

It follows that $v$ is an injection.

We claim that $v$ is an isomorphism. Since $H_{*}(\Omega Y ; R)$ is torsion-free, it has the same Hilbert series as $H_{*}(\Omega Y ; \mathbb{Q})$. Let $S$ be the image of $h_{Y} \otimes \mathbb{Q}$. Then $S$ and $L_{Y}$ have the same Hilbert series. By the Milnor-Moore Theorem [MM65], $H_{*}(\Omega Y ; \mathbb{Q}) \cong U S$. So by the Poincaré-Birkhoff-Witt Theorem, $S$ has the same Hilbert series as $\hat{L}$, and hence $L_{Y} \cong \hat{L}$ as $R$-modules. Since $v: \hat{L} \rightarrow L_{Y}$ is an injection, it follows that it is an isomorphism.

Therefore $L_{Y} \cong L_{Y}^{X} \amalg \mathbb{L} \hat{K}$ as Lie algebras, with $\hat{K} \subset F_{1} L_{Y}$ and $H_{*}(\Omega Y ; R) \cong$ $U L_{Y}$ as algebras.

Proof of Theorem 5.5(c). Lemma 5.6 proves (i) and puts us in a position to prove (ii) and (iii).

(ii) Recall that $(H \underline{\mathbf{L}})_{1} \cong \hat{K}$ and $L^{\prime}=(H \underline{\mathbf{L}})_{0} \amalg \mathbb{L}\left((H \underline{\mathbf{L}})_{1}\right)$. Thus there is a canonical map $\nu: L^{\prime} \rightarrow H \underline{\mathbf{L}}$. Now the composition

$$
L^{\prime} \stackrel{\nu}{\rightarrow} H \underline{\mathbf{L}} \stackrel{\phi}{\rightarrow} H U \underline{\mathbf{L}} \stackrel{\cong}{\rightarrow} U L^{\prime}
$$


is just the ordinary inclusion of $L^{\prime}$ into its universal enveloping algebra. Therefore $\nu$ is an injection. Tensoring with $\mathbb{Q}$ we get that $L^{\prime}$ and $H \underline{\mathbf{L}}$ have the same Hilbert series. It follows that $\nu$ is an isomorphism.

(iii) We will construct a map $\sigma_{Y}$ right inverse to $h_{Y}$.

Let $i$ denote the inclusion $X \hookrightarrow Y$. Consider the composite map

$$
F:\left[L_{X}^{W}\right] \hookrightarrow L_{X} \stackrel{\sigma_{X}}{\longrightarrow} \pi_{*}(\Omega X) \otimes R \stackrel{(\Omega i)_{\#}}{\longrightarrow} \pi_{*}(\Omega Y) \otimes R .
$$

We claim that $F=0$. Since $F$ is a Lie algebra map it is sufficient to show that it is zero on the Lie algebra generators of $L_{X}^{W}$. That is, show

$$
(\Omega i)_{\#} \sigma_{X}\left(R\left\{h_{X}\left(\hat{\alpha}_{j}\right)\right\}\right)=0 .
$$

Since there are no implicit primes $\sigma_{X} h_{X} \hat{\alpha}_{j}=\hat{\alpha}_{j}$. By the construction of $Y, \Omega i \circ \hat{\alpha}_{j} \simeq$ 0 . So $F=0$ as claimed.

Therefore there is an induced map $G: L_{Y}^{X} \cong L_{X} /\left[L_{X}^{W}\right] \rightarrow \pi_{*}(\Omega Y) \otimes R$. That $h_{Y} \circ G$ is the inclusion map can be seen from the following commutative diagram:

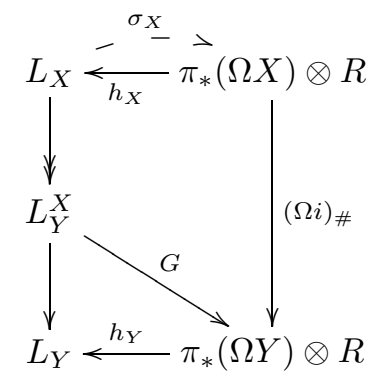

Now construct $\sigma_{Y}: L_{Y} \rightarrow \pi_{*}(\Omega Y) \otimes R$ as follows. We have shown that $L_{Y} \cong$ $L_{Y}^{X} \amalg \mathbb{L} \hat{K}$ for some $\hat{K} \subset F_{1} L_{Y}$. Since $h_{Y}: \pi_{*}(\Omega Y) \otimes R \rightarrow L_{Y}$, choose preimages $\check{K} \subset \pi_{*}(\Omega Y) \otimes R$ such that $h_{Y}: \check{K} \cong \hat{K}$. Let $\left.\sigma_{Y}\right|_{L_{Y}^{X}}=G$ and let $\sigma_{Y} \hat{K}=\check{K}$ be right inverse to $h_{Y}$. Now extend $\sigma_{Y}$ canonically to a Lie algebra map on $L_{Y}$.

We finally claim that $h_{Y} \sigma_{Y}=i d_{L_{Y}}$. Since $h_{Y} \sigma_{Y}$ is a Lie algebra map it suffices to check that it is the identity for the generators

$$
h_{Y} \sigma_{Y} L_{Y}^{X}=h_{Y} G L_{Y}^{X}=L_{Y}^{X}, \quad h_{Y} \sigma_{Y} \hat{K}=h_{Y} \check{K}=\hat{K} .
$$

Therefore $\sigma_{Y}$ is the desired Lie algebra map right inverse to $h_{Y}$.

Remark 5.7. Anick conjectured Theorem 5.5(c)(ii) without the semi-inert condition in the special case where $X$ is a wedge of spheres Ani89, Conj. 4.9].

Corollary 5.8 (Corollary 1.8). If $R \subset \mathbb{Q}$, then the canonical algebra map

$$
U\left(F\left(\pi_{*}(\Omega Y) \otimes R\right)\right) \rightarrow H_{*}(\Omega Y ; R)
$$

is an isomorphism.

Proof. Recall that $F M=M / \operatorname{Torsion}(M)$. By definition, the Lie algebra map $h_{Y}: \pi_{*}(\Omega Y) \otimes R \rightarrow L_{Y}$ is a surjection. By Theorem 4.3, $L_{Y} \subset H_{*}(\Omega Y ; R)$ is torsion-free. As a result, there is an induced surjection $\tilde{h}_{Y}: F\left(\pi_{*}(\Omega Y) \otimes R\right) \rightarrow L_{Y}$.

Furthermore if we tensor with $\mathbb{Q}$ we see that $\pi_{*}(\Omega Y) \otimes \mathbb{Q} \stackrel{\cong}{\rightrightarrows} L_{Y} \otimes \mathbb{Q}$, which is a result of Cartan and Serre (see [FHT01]). Thus $F\left(\pi_{*}(\Omega Y) \otimes R\right)$ and $L_{Y}$ have the same Hilbert series, and therefore $\tilde{h}_{Y}$ is an isomorphism of Lie algebras. 
So using Theorem [5.5(b), the canonical algebra map

$$
U\left(F\left(\pi_{*}(\Omega Y) \otimes R\right)\right) \rightarrow U L_{Y} \cong H_{*}(\Omega Y ; R)
$$

is an isomorphism.

Corollaries 1.9 and 1.10 follow immediately from Theorem 5.5.

\section{EXAmples}

(Spherical) $n$-cones are those spaces $X$ such that there exists a finite sequence

$$
*=X_{0} \subset X_{1} \subset \cdots \subset X_{n} \simeq X
$$

where for $k \geq 0, X_{k+1}$ is the adjunction space

$$
X_{k+1}=X_{k} \cup_{f_{k+1}}\left(\bigvee_{j \in J} e^{n_{j}+1}\right) .
$$

In particular, any finite $\mathrm{CW}$-complex is an $n$-cone for some $n$.

Example 6.1. Let $X=S_{a}^{3} \vee S_{b}^{3}$ and let $\iota_{a}, \iota_{b}$ denote the inclusions of the spheres into $X$. Let $Y=X \cup_{\alpha_{1} \vee \alpha_{2}}\left(e^{8} \vee e^{8}\right)$, where the attaching maps are given by the iterated Whitehead products $\alpha_{1}=\left[\left[\iota_{a}, \iota_{b}\right], \iota_{a}\right]$ and $\alpha_{2}=\left[\left[\iota_{a}, \iota_{b}\right], \iota_{b}\right]$.

Let $W=S^{7} \vee S^{7}$ and for $i=1,2$ let $\hat{\alpha}_{i}: S^{6} \rightarrow \Omega X$ denote the adjoint of $\alpha_{i}$. Let $R=\mathbb{F}_{p}$ with $p>3$ or $R \subset \mathbb{Q}$ containing $\frac{1}{6}$. Then $\left[L_{X}^{W}\right]=\left[R\left\{h_{X}\left(\hat{\alpha}_{1}\right), h_{X}\left(\hat{\alpha}_{2}\right)\right\}\right]$.

$Y$ has an Adams-Hilton model (see Section 2) $U(L, d)$, where $L=\mathbb{L}\langle x, y, a, b\rangle,|x|$ $=|y|=2, d x=d y=0, d a=[[x, y], x]$ and $d b=[[x, y], y]$. Furthermore $h_{X}\left(\hat{\alpha}_{1}\right)=$ $[d a]$ and $h_{X}\left(\hat{\alpha}_{2}\right)=[d b]$.

It is well known that over a field, a Lie subalgebra of a free Lie algebra is also free. Thus, since $\mathbb{L}\langle x, y\rangle$ is a free Lie algebra, $U L$ is a free dga extension (in the sense of Definition 3.9).

Let $u=[a, y]-[b, x]$. Then $d u=[[[x, y], x], y]-[[[x, y], y], x]=[[x, y],[x, y]]=0$. Since $u$ is not a boundary, $0 \neq[u] \in(H L)_{1}$ and $0 \neq[u] \in(H U L)_{1}$. Thus $U L$ is not an inert dga extension. By the definition of homology

$$
(H L)_{0} \cong \mathbb{L}\langle x, y\rangle /[R\{[[x, y], x],[[x, y], y]\}] .
$$

One can check that $(H L)_{1}$ is freely generated by the $(H L)_{0}$-action on $[u]$. Thus $U L$ is a semi-inert extension. Therefore by Theorem 3.12(b),

$$
H U L \cong U\left((H L)_{0} \amalg \mathbb{L}\langle[u]\rangle\right)
$$

as algebras. Thus as algebras

$$
\begin{aligned}
H_{*}(\Omega Y ; R) & \cong U\left(L_{X} /\left[L_{X}^{W}\right] \amalg \mathbb{L}\langle[u]\rangle\right) \\
& \cong\left(H_{*}(\Omega X ; R) /\left(L_{X}^{W}\right)\right) \amalg \mathbb{T}\langle[u]\rangle .
\end{aligned}
$$

$\alpha_{1} \vee \alpha_{2}$ is a non-inert, semi-inert attaching map.

If $R=\mathbb{Z}\left[\frac{1}{6}\right]$, then since $X$ is a wedge of spheres, there exists a map $\sigma_{X}$ right inverse to $h_{X}$. By Lemma [5.2, $P_{Y}=\{2,3\}$. By Theorem [5.5,

$$
H_{*}(\Omega Y ; R) \cong U L_{Y}, \quad L_{Y} \cong L_{Y}^{X} \amalg \mathbb{L}\langle w\rangle \cong \mathbb{L}\langle x, y, w\rangle / J,
$$

where $w=h_{Y}(\hat{\omega})$ with $\hat{\omega}$ the adjoint of some map $\omega: S^{10} \rightarrow Y$, and $J$ is the Lie ideal generated by $\{[[x, y], x],[[x, y], y]\}$. Furthermore localized at $\mathrm{R}, \Omega Y \in \prod \mathcal{S}$ and there exists a map $\sigma_{Y}$ right inverse to $h_{Y}$. 
Example 6.2. The 6-skeleton of $S^{3} \times S^{3} \times S^{3}$.

This space $Y$ is also known as the fat wedge $F W\left(S^{3}, S^{3}, S^{3}\right)$. Let $X=S_{a}^{3} \vee S_{b}^{3} \vee$ $S_{c}^{3}$. Let $\iota_{a}, \iota_{b}$ and $\iota_{c}$ be the inclusions of the respective spheres. Let $W=\bigvee_{j=1}^{3} S^{5}$. Then $Y=X \cup_{f}\left(\bigvee_{j=1}^{3} e_{j}^{6}\right)$, where $f: W \rightarrow X$ is given by $\bigvee_{j=1}^{3} \alpha_{j}$ with $\alpha_{1}=\left[\iota_{b}, \iota_{c}\right]$, $\alpha_{2}=\left[\iota_{c}, \iota_{a}\right]$ and $\alpha_{3}=\left[\iota_{a}, \iota_{b}\right]$.

Let $R=\mathbb{Z}\left[\frac{1}{6}\right]$. Then $Y$ has the Adams-Hilton model $U(L, d)$, where $L=$ $\mathbb{L}\langle x, y, z, a, b, c\rangle,|x|=|y|=|z|=2, d x=d y=d z=0, d a=[y, z], d b=[z, x]$ and $d c=[x, y]$. Let $w=[x, a]+[y, b]+[z, c]$.

By the same argument as in the previous example, as algebras

$$
H_{*}(\Omega Y ; R) \cong U L_{Y}, \quad L_{Y} \cong L_{Y}^{X} \amalg \mathbb{L}\langle w\rangle \cong \mathbb{L}\langle x, y, z, w\rangle / J,
$$

where $w=h_{Y}(\hat{\omega})$ with $\hat{\omega}$ the adjoint of some map $\omega: S^{8} \rightarrow Y$ and $J$ is the Lie ideal generated by $\{[x, y],[y, z],[z, x]\}$. Furthermore localized at $R, \Omega Y \in \prod \mathcal{S}$ and there exists a map $\sigma_{Y}$ right inverse to $h_{Y}$.

The following spherical three-cone $Y$ illustrates our results.

Example 6.3. Let $R=\mathbb{Z}\left[\frac{1}{6}\right]$. All spaces here are localized at $R$. For $i=1,2$ let $Z_{i}=A_{i} \cup_{\alpha_{1} \vee \alpha_{2}}\left(e^{8} \vee e^{8}\right)$ be two copies of the two-cone from Example 6.1, Let $X=Z_{1} \vee Z_{2}, W=S^{28} \vee S^{28}$ and let $f=\beta_{1} \vee \beta_{2}$, where $\beta_{1}=\left[\left[\omega_{1}, \omega_{2}\right], \omega_{1}\right]$ and $\beta_{2}=\left[\left[\omega_{1}, \omega_{2}\right], \omega_{2}\right]$. Let $Y=X \cup_{f}\left(e^{29} \vee e^{29}\right)$.

Now,

$$
L_{X} \cong L_{Z_{1}} \amalg L_{Z_{2}} \cong L_{Z_{1}}^{A_{1}} \amalg L_{Z_{2}}^{A_{2}} \amalg \mathbb{L}\left\langle w_{1}, w_{2}\right\rangle .
$$

If follows from this that $f$ is a free attaching map. Thus $Y$ satisfies the hypotheses of Theorem 4.3. Using Theorem 4.3 and Anick's formula, one can calculate that if $f$ is semi-inert, then $K^{\prime}(z)=z^{37}$.

Recall that $\underline{\mathbf{L}}=\left(L_{X} \amalg \mathbb{L}\langle e, g\rangle, d^{\prime}\right)$, where $d^{\prime} e=\left[\left[w_{1}, w_{2}\right], w_{1}\right]$ and $d^{\prime} g=\left[\left[w_{1}, w_{2}\right]\right.$, $\left.w_{2}\right]$. Also recall (from Theorem $[.3)$ that $(H \underline{\mathbf{L}})_{0} \cong L_{Y}^{X}$. Let $\bar{u}=\left[e, w_{2}\right]+\left[g, w_{1}\right]$ (with $|\bar{u}|=37$ ). Then one can check that $d \bar{u}=0$ and $[\bar{u}]$ is a basis for $(H \underline{\mathbf{L}})_{1}$ as a free $(H \underline{\mathbf{L}})_{0}$-module; so $f$ is indeed semi-inert. Let $\sigma_{X}=\sigma_{Z_{1}} \oplus \sigma_{Z_{2}}$. It is right inverse to $h_{X}$. By Lemma 5.2, $P_{Y}=\{2,3\}$. As a result by Theorem5.5,

$$
\begin{gathered}
H_{*}(\Omega Y ; R) \cong U L_{Y}, \text { where } \\
L_{Y} \cong L_{Y}^{X} \amalg \mathbb{L}\langle u\rangle \cong \mathbb{L}\left\langle x_{1}, y_{1}, x_{2}, y_{2}, w_{1}, w_{2}, u\right\rangle / J,
\end{gathered}
$$

with $u=h_{Y}(\hat{\mu})$ for some map $\mu: S^{38} \rightarrow Y$ and $J$ the Lie ideal generated by

$$
\begin{array}{r}
\left\{\left[\left[x_{1}, y_{1}\right], x_{1}\right],\left[\left[x_{1}, y_{1}\right], y_{1}\right],\left[\left[x_{2}, y_{2}\right], x_{2}\right],\right. \\
\left.\left[\left[x_{2}, y_{2}\right], y_{2}\right],\left[\left[w_{1}, w_{2}\right], w_{1}\right], \quad\left[\left[w_{1}, w_{2}\right], w_{2}\right]\right\} .
\end{array}
$$

Furthermore $\Omega Y \in \prod \mathcal{S}$ and there exists a map $\sigma_{Y}$ right inverse to $h_{Y}$.

Note that

$$
L_{Y} \cong L_{1}^{1} \amalg L_{1}^{2} \amalg L_{2} \amalg \mathbb{L}\langle u\rangle,
$$

where $L_{1}^{1} \cong L_{1}^{2} \cong R\{x, y,[x, y]\}$ and $L_{2} \cong R\left\{w_{1}, w_{2},\left[w_{1}, w_{1}\right],\left[w_{1}, w_{2}\right],\left[w_{2}, w_{2}\right]\right\}$. 
Example 6.4. An infinite family of finite CW-complexes constructed out of semiinert attaching maps.

The construction in the previous example can be extended inductively. By induction, we will construct spaces $X_{n}$ and maps $\omega_{n}: S^{\lambda_{n}} \rightarrow X_{n}$ for $n \geq 1$ such that $X_{n}$ is an $n$-cone constructed out of a sequence of semi-inert attaching maps. Given $\omega_{n}$, let $w_{n}=h_{X_{n}}\left(\left[\omega_{n}\right]\right)$ and given $w_{i}^{a}$ and $w_{i}^{b}$, let $L_{i}=\mathbb{L}\left\langle w_{i}^{a}, w_{i}^{b}\right\rangle / J_{i}$, where $J_{i}$ is the Lie ideal of brackets of bracket length $\geq 3$.

Let $R=\mathbb{Z}\left[\frac{1}{6}\right]$. Begin with $X_{1}=S^{3}$ and $\lambda_{1}=3$. Let $\omega_{1}: S^{\lambda_{1}} \rightarrow X_{1}$ be the identity map.

Given $X_{n}$, let $X_{n}^{a}$ and $X_{n}^{b}$ be two copies of $X_{n}$. For $n \geq 1$, let

$$
X_{n+1}=X_{n}^{a} \vee X_{n}^{b} \cup_{f_{n+1}}\left(e^{\kappa_{n+1}} \vee e^{\kappa_{n+1}}\right),
$$

where $\kappa_{n+1}=3 \lambda_{n}-1$ and $f_{n+1}=\left[\left[\omega_{n}^{a}, \omega_{n}^{b}\right], \omega_{n}^{a}\right] \vee\left[\left[\omega_{n}^{a}, \omega_{n}^{b}\right], \omega_{n}^{b}\right]$.

By the same argument as in the previous example, $f_{n+1}$ is a semi-inert cell attachment and there exists a map

$$
\omega_{n+1}: S^{\lambda_{n+1}} \rightarrow X_{n+1},
$$

where $\lambda_{n+1}=4 \lambda_{n}-2$, such that

$$
H_{*}\left(\Omega X_{n+1} ; R\right) \cong U L_{X_{n+1}}, \text { where } L_{X_{n+1}}=\left(\coprod_{\substack{1 \leq i \leq n \\ 1 \leq j \leq 2^{n-i}}} L_{i}^{j}\right) \amalg \mathbb{L}\left\langle w_{n+1}\right\rangle
$$

with $L_{i}^{j}$ a copy of $L_{i}$ and $w_{n+1}=h_{X_{n+1}}\left(\hat{\omega}_{n+1}\right)$.

Example 6.5. An uncountable family of CW-complexes constructed out of semiinert cell attachments.

At each stage of the inductive construction in Example 6.4 we could have used an attachment of the type in Example6.2 instead of an attachment of the type in Example 6.1] For $\alpha \in[0,1)$ use the binary expansion of $\alpha$ to choose the sequence of attachments to obtain a space $X_{\alpha}$.

Example 6.6. CW-complexes $X$ with only odd-dimensional cells.

Let $X^{(n)}$ denote the $n$-skeleton of $X$. From the CW-structure of $X$, there is a sequence of cell attachments for $k \geq 1$.

$$
W_{2 k} \stackrel{f_{2 k+1}}{\longrightarrow} X^{(2 k-1)} \rightarrow X^{(2 k+1)},
$$

where $W_{2 k}$ is a finite wedge of $(2 k)$-dimensional spheres, $X^{(2 k+1)}$ is the adjunction space of the cell attachment and $X^{(1)}=*$. Assume $R \subset \mathbb{Q}$ containing $\frac{1}{6}$. Let $P_{X^{(n)}}$ be the set of implicit primes of $X^{(n)}$ (see Definition [5.1). We will show by induction that

$$
H_{*}\left(\Omega X^{(2 k+1)} ; \mathbb{Z}\left[P_{X^{(2 k+1)}}^{-1}\right]\right) \cong U L_{X^{(2 k+1)}},
$$

where $L_{X^{(2 k+1)}} \cong \mathbb{L} V^{(2 k+1)}$ with $V^{(2 k+1)}$ concentrated in even dimensions. In addition localized away from $P_{X^{(2 k+1)}}, \Omega X^{(2 k+1)} \in \prod \mathcal{S}$ and there exists a map $\sigma_{X^{(2 k+1)}}$ right inverse to $h_{X^{(2 k+1)}}$.

For $k=0$ these conditions are trivial. Assume they hold for $k-1$. Let $\underline{\mathbf{L}}=\left(L_{X^{(2 k-1)}} \amalg \mathbb{L} K, d^{\prime}\right)$, where $K$ is a free $R$-module in dimension $2 k$ corresponding to the spheres in $W_{2 k}$. For degree reasons $L_{X^{(2 k-1)}}^{W_{2 k}}=d^{\prime}(K)=0$. So $f_{2 k+1}$ is automatically free. Furthermore $\underline{\mathbf{L}}$ has zero differential so $H \underline{\mathbf{L}}=\mathbb{L} V^{(2 k-1)} \amalg \mathbb{L} K$. Thus 
$f_{2 k+1}$ is semi-inert. By Theorem $5.5 H_{*}\left(\Omega X^{(2 k+1)} ; \mathbb{Z}\left[P_{X^{(2 k+1)}}{ }^{-1}\right]\right) \cong U L_{X^{(2 k+1)}}$, where

$$
L_{X^{(2 k+1)}} \cong L_{X^{(2 k+1)}}^{X^{(2 k-1)}} \amalg \mathbb{L} K \cong L_{X^{(2 k-1)}} \amalg \mathbb{L} K \cong \mathbb{L}\left(V^{(2 k-1)} \oplus K\right) .
$$

Also by Theorem 5.5, localized away from $P_{X^{(2 k+1)}}, \Omega X^{(2 k+1)} \in \prod \mathcal{S}$, and there exists a map $\sigma_{X^{(2 k+1)}}$ right inverse to $h_{X^{(2 k+1)}}$.

Therefore by induction $H_{*}\left(\Omega X ; \mathbb{Z}\left[P_{X}{ }^{-1}\right]\right) \cong U L_{X}$, where $L_{X} \cong \mathbb{L}\left(s^{-1} \tilde{H}_{*}(X)\right)$ with $s$ the suspension map, and localized away from $P_{X}, \Omega X \in \prod \mathcal{S}$.

\section{ACKNOWLEDGMENTS}

The results of this paper were part of my thesis at the University of Toronto Bub03. I would like to thank my advisor, Paul Selick, for his encouragement and support, and Jean-Michel Lemaire for his helpful suggestions.

\section{REFERENCES}

[AH56] J. F. Adams and P. J. Hilton, On the chain algebra of a loop space, Comment. Math. Helv. 30 (1956), 305-330. MR0077929 (17:1119b)

[Ani82] David J. Anick, A counterexample to a conjecture of Serre, Ann. of Math. (2) 115 (1982), no. 1, 1-33. MR0644015 (86i:55011a)

[Ani89] Homotopy exponents for spaces of category two, Algebraic topology (Arcata, CA, 1986), Lecture Notes in Math., vol. 1370, Springer, Berlin, 1989, pp. 24-52. MR.1000365 (90c:55010)

[Ani92] , Single loop space decompositions, Trans. Amer. Math. Soc. 334 (1992), no. 2, 929-940. MR1145728 (93g:55011)

[Avr82] Luchezar L. Avramov, Free Lie subalgebras of the cohomology of local rings, Trans. Amer. Math. Soc. 270 (1982), no. 2, 589-608. MR0645332 (83g:13010)

[Bau81] Hans Joachim Baues, Commutator calculus and groups of homotopy classes, London Mathematical Society Lecture Note Series, vol. 50, Cambridge University Press, Cambridge, 1981. MR 0634675 (83b:55012)

[Bub03] Peter Bubenik, Cell attachments and the homology of loop spaces and differential graded algebras, Ph.D. thesis, University of Toronto, 2003.

[Bub04] , Free cell attachments and the rational homotopy lie algebra, arXiv:math.AT/0406405, 2004.

[FHT84] Yves Félix, Stephen Halperin, and Jean-Claude Thomas, Sur l'homotopie des espaces de catégorie 2, Math. Scand. 55 (1984), no. 2, 216-228. MR0787198 (86k:55006)

[FHT01] _ Rational homotopy theory, Graduate Texts in Mathematics, vol. 205, SpringerVerlag, New York, 2001. MR1802847 (2002d:55014)

[FT89] Yves Félix and Jean-Claude Thomas, Effet d'un attachement cellulaire dans l'homologie de l'espace des lacets, Ann. Inst. Fourier (Grenoble) 39 (1989), no. 1, 207-224. MR.1011984 (90j:55012)

[HL87] Stephen Halperin and Jean-Michel Lemaire, Suites inertes dans les algèbres de Lie graduées ("Autopsie d'un meurtre. II"), Math. Scand. 61 (1987), no. 1, 39-67. MR.0929396 (89e:55022)

[HL96] Kathryn Hess and Jean-Michel Lemaire, Nice and lazy cell attachments, J. Pure Appl. Algebra 112 (1996), no. 1, 29-39. MR1402394 (97e:55006)

[Lem78] Jean-Michel Lemaire, "Autopsie d'un meurtre" dans l'homologie d'une algèbre de châ̂nes, Ann. Sci. École Norm. Sup. (4) 11 (1978), no. 1, 93-100. MR0500930|(58:18423)

[MM65] John W. Milnor and John C. Moore, On the structure of Hopf algebras, Ann. of Math. (2) 81 (1965), 211-264. MR0174052 (30:4259)

[Sco02] Jonathan A. Scott, Algebraic structure in the loop space homology Bockstein spectral sequence, Trans. Amer. Math. Soc. 354 (2002), no. 8, 3075-3084 (electronic). MR1897391 (2003c:55008)

[Sco03] , A torsion-free Milnor-Moore theorem, J. London Math. Soc. (2) 67 (2003), no. 3, 805-816. MR 1967707 (2004c:55017) 
[Whi39] J. H. C. Whitehead, Simplicial spaces, nuclei and m-groups, Proc. London Math. Soc. (2) 45 (1939), 243-327.

[Whi41] - On adding relations to homotopy groups, Ann. of Math. (2) 42 (1941), 409-428. MR0004123 (2:323c)

Institut de Géométrie, Algèbre et Topologie, Ecole Polytechnique Fédérale de Lausanne, EPFL/SB/IGAT (BCH), 1015 Lausanne, Switzerland

E-mail address: peter.bubenik@epfl.ch 